\title{
DIFFERENTIAL OPERATORS ON AN AFFINE CURVE: IDEAL CLASSES AND PICARD GROUPS
}

\author{
YURI BEREST AND GEORGE WILSON
}

\begin{abstract}
Let $X$ be a smooth complex affine curve, and let $\mathcal{R}$ be the space of right ideal classes in the ring $\mathcal{D}$ of differential operators on $X$. We introduce and study a fibration $\gamma: \mathcal{R} \rightarrow \operatorname{Pic} X$. We relate this fibration to the corresponding one in the classical limit, and derive an integer invariant $n$ which indexes the decomposition of the fibres of $\gamma$ into Calogero-Moser spaces (see $[\mathrm{BC}]$ ). We also study the action of the group Pic $\mathcal{D}$ on our fibration; and we explain how to define $\gamma$ in the framework of the Grassmannian description of $\mathcal{R}$ due to Cannings and Holland.
\end{abstract}

\section{INTRODUCTION}

Let $X$ be an irreducible smooth affine algebraic curve over $\mathbb{C}$, and let $\mathcal{D}$ be the ring of differential operators on $\mathcal{O}(X)$. We denote by $\mathcal{R}$ the set of isomorphism classes of right ideals in $\mathcal{D}$ (equivalently: of finitely generated torsion-free right $\mathcal{D}$-modules of rank 1 ). In the case $X=\mathbb{A}^{1}$, where $\mathcal{D}$ is the Weyl algebra, $\mathcal{R}$ can be described in two essentially different ways (i) as the adelic Grassmannian of W1 (see [CH1); (ii) as the disjoint union of certain finite-dimensional algebraic varieties, the Calogero-Moser spaces $\mathcal{C}_{n}$ (see [BW1, BW2]). The general case is similar (see $[\mathrm{BC}, \overline{\mathrm{Be}}, \mathrm{W} 3, \overline{\mathrm{BN}}$ ); however, some new features come into play. In this preliminary paper we make a fairly thorough study of one of them, which comes from the fact that if $X$ has genus $>0$ then the Picard group Pic $X$ is nontrivial. We recall that Pic $X$ is the group of algebraic line bundles (or divisor classes) over $X$; alternatively, we may think of it as the group of ideal classes in the Dedekind domain $\mathcal{O}(X)$. We shall see that there is a natural fibration

$$
\gamma: \mathcal{R} \rightarrow \operatorname{Pic} X \text {. }
$$

The significance of this fibration is that it is the individual fibres of $\gamma$ (rather than the whole of $\mathcal{R}$ ) that have descriptions generalizing those already known in the case $X=\mathbb{A}^{1}$. Of course, in that case Pic $X$ is trivial, so there is only one fibre.

The definition of $\gamma$ is via $K$-theory: if we wish to classify the ideals in $\mathcal{D}$ up to isomorphism, we should certainly understand first how to classify them up to stable isomorphism. Let $K_{0}(\mathcal{D})$ be the Grothendieck group of finitely generated projective right $\mathcal{D}$-modules: since $\mathcal{D}$ is Noetherian and hereditary, every ideal $M \subseteq \mathcal{D}$ is finitely generated and projective, hence determines a class $[M] \in K_{0}(\mathcal{D})$. The inclusion of $\mathcal{O}(X)$ in $\mathcal{D}$ as the operators of order zero induces a map from $K_{0}(X)$ $\left(\equiv K_{0}(\mathcal{O}(X))\right)$ to $K_{0}(\mathcal{D})$; a theorem of Quillen (see Q, p. 120, Theorem 7) shows that this map is an isomorphism. On the other hand, it is well known that the map

$$
\operatorname{rk} \oplus \operatorname{det}: K_{0}(X) \rightarrow \mathbb{Z} \oplus \operatorname{Pic} X
$$


is also an isomorphism. Since $\operatorname{rk} M=1$, it follows that the stable isomorphism class of $M$ is determined by its component in Pic $X$ : we define $\gamma(M)$ to be this component. That is, if $M$ is a right ideal in $\mathcal{D}$, then $\gamma(M)$ is the unique ideal class $(I) \in \operatorname{Pic} X$ such that $M$ is stably isomorphic to ID.

Now, as for any algebra $\mathcal{D}$, the group Pic $\mathcal{D}$ of invertible $\mathcal{D}$-bimodules acts (compatibly) on modules and on $K_{0}(\mathcal{D})$ by tensor product. Thus we have natural actions of $\mathrm{Pic} \mathcal{D}$ on $\mathcal{R}$ and on $\mathrm{Pic} X$, and the map $\gamma$ is equivariant with respect to these actions. The following fact perhaps justifies referring to $\gamma$ as a "fibration".

Theorem 1.1. The action of Pic $\mathcal{D}$ on $\mathrm{Pic} X$ is transitive. Thus for any two fibres $F_{1}$ and $F_{2}$ of $\gamma$ there are elements of the group Pic $\mathcal{D}$ that map $F_{1}$ bijectively onto $F_{2}$.

Next, we wish to compare our situation with the corresponding one in the "classical limit". Recall that if we give $\mathcal{D}$ its usual filtration by order of operators, then the associated graded algebra $\overline{\mathcal{D}}:=\operatorname{gr} \mathcal{D}$ is canonically identified with the commutative algebra $\mathcal{O}\left(T^{*} X\right)$ of functions on the cotangent bundle of $X$. Thus our space $\mathcal{R}$ is a noncommutative analogue of the space $\overline{\mathcal{R}}$ of rank 1 torsion-free sheaves on $T^{*} X$, or (since $T^{*} X$ is affine if $X$ is) of ideal classes in $\overline{\mathcal{D}}$. If $M$ is an ideal in $\mathcal{D}$, we may give it the filtration induced by that of $\mathcal{D}$; the associated graded module $\bar{M}:=\operatorname{gr} M$ is then a (homogeneous) ideal in $\overline{\mathcal{D}}$, and we obtain a well-defined map gr $: \mathcal{R} \rightarrow \overline{\mathcal{R}}$. There is also a map $\bar{\gamma}: \overline{\mathcal{R}} \rightarrow \operatorname{Pic} X$, which can be defined in several ways (see Proposition 2.1 below). As the basic one, we choose the following. Let $F$ be an ideal in $\overline{\mathcal{D}}$, and let $F^{*}$ be the dual $\overline{\mathcal{D}}$-module. Although $F$ is in general not locally free, $F^{*}$ is; a fortiori, the bidual $F^{* *}$ is locally free, so defines an element of $\operatorname{Pic}\left(T^{*} X\right)$. The projection $p: T^{*} X \rightarrow X$ induces an isomorphism $\operatorname{Pic} X \cong \operatorname{Pic}\left(T^{*} X\right)$ : we define $\bar{\gamma}(F)$ to be the class in Pic $X$ corresponding to $\left(F^{* *}\right)$ under this isomorphism.

Theorem 1.2. The diagram

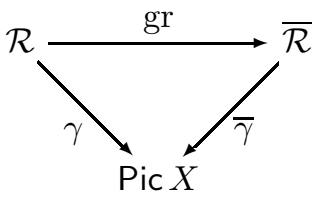

commutes.

In contrast to our noncommutative case, the fibration $\bar{\gamma}$ has a natural trivialization: in fact each fibre of $\bar{\gamma}$ is canonically identified with the disjoint union of the point Hilbert schemes $\operatorname{Hilb}_{n}\left(T^{*} X\right)$ for $n \geq 0$ : see Section 2 for more details.

It is a general property of surfaces (we are concerned with the case $T^{*} X$ ) that the canonical inclusion $F \hookrightarrow F^{* *}$ of an ideal of $\overline{\mathcal{D}}$ in its bidual has finite codimension. This fact gives us an important invariant $n=n(M)$ of an ideal (class) $M$ in $\mathcal{D}$ : namely, $n(M)$ is the codimension of $\bar{M}$ in its bidual. As we shall see in [BC], this invariant $n$ indexes the Calogero-Moser stratum $\mathcal{C}_{n}$ to which $M$ belongs in the decomposition of the fibres of $\gamma$. In the present paper we content ourselves with proving the following.

Theorem 1.3. The invariant $n$ is constant on the orbits of $\operatorname{Pic} \mathcal{D}$ in $\mathcal{R}$.

The last theorem we want to formulate in this Introduction makes contact with the description of $\mathcal{R}$ given in [CH1], which (in contrast to the above) seems to have 
no classical analogue. To each ideal class $(M) \in \mathcal{R}$ Cannings and Holland assign a primary decomposable subspace $V$ of $\mathcal{O}(X)$, well defined up to multiplication by a rational function. We review the precise definition in Section 5] here we just recall that $V$ is determined by a finite set of points $x_{1}, \ldots, x_{k}$ of $X$, together with a subspace $V_{i} \subset \mathcal{O}(X)$ associated to each point $x_{i}$. Each $V_{i}$ has finite codimension, say $n_{i}$, in $\mathcal{O}(X)$. Thus we may assign to $V$ the divisor

$$
\operatorname{Div}(V):=-\sum_{i=1}^{k} n_{i} x_{i}
$$

(the bizarre minus sign is explained by Theorem 1.4 below). If we multiply $V$ by a rational function, the class of $\operatorname{Div}(V)$ in Pic $X$ stays the same: assigning to $(M)$ this divisor class, we get another map

$$
\text { Div }: \mathcal{R} \rightarrow \text { Pic } X \text {. }
$$

Theorem 1.4. The map (1.3) coincides with $\gamma$.

The paper is arranged as follows. In Section 2 we discuss briefly the the commutative analogue of our problem and give the proof of Theorem 1.2 , Section 3 is devoted to the action of Pic $\mathcal{D}$, and contains the proof of Theorems 1.1 and 1.3 Section 4 could be read in conjunction with Section 2 it contains a more refined version of $\gamma$ at the level of ideals (rather than ideal classes). That is needed in the last section, which gives the proof of Theorem 1.4. Finally, the two appendices provide proofs for some of the less well known facts used in Section 2

To end this introduction, we point out that our choice to work with right ideals in this paper was quite arbitrary, and that there are similar results for the space $\mathcal{J}$ of left ideals. Indeed, the dualization map $M \mapsto \operatorname{Hom}_{\mathcal{D}}(M, \mathcal{D})$ induces a natural bijection $\delta: \mathcal{R} \rightarrow \mathcal{J}$, and the inclusion of $\mathcal{O}(X)$ into $\mathcal{D}$ still identifies the Grothendieck group of finitely generated left projective $\mathcal{D}$-modules with $\mathbb{Z} \oplus$ Pic $X$. It follows that we have a commutative diagram

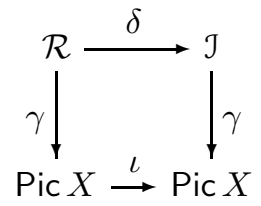

where $\iota$ is the inverse operation $I \mapsto I^{*}$ in the group Pic $X$. Using this diagram we can deduce results for left ideals from those for right ideals without rewriting our whole paper.

Notation. As above, if $V$ is a filtered vector space, the associated graded space $\operatorname{gr} V$ will often be denoted by $\bar{V}$; and if $M$ is a module over a ring, we shall write $[M]$ for the class of $M$ in the appropriate Grothendieck group, and (if necessary) $(M)$ for the isomorphism class of $M$. To avoid fussy notation we will sometimes confuse $M$ and $(M)$. We denote by $\mathbb{K}$ the quotient field of $\mathcal{O}(X)$ and by $\mathcal{D}(\mathbb{K})$ the ring of differential operators on $\mathbb{K}$.

\section{The Commutative Case}

General remarks. Recall first that if $\mathcal{F}$ is a torsion-free coherent sheaf over any smooth variety, the singular set 11 of $\mathcal{F}$ has codimension at least 2 , and if $\mathcal{F}$

\footnotetext{
${ }^{1}$ That is, the set of points near which $\mathcal{F}$ is not locally free.
} 
is reflexive its singular set has codimension at least 3 (see, for example OSS, Corollary, p. 148 and Theorem 1.1.6, p. 145). In the case of a smooth surface $S$, these simple facts already yield a lot of information about the space $\mathcal{R}(S)$ of rank 1 torsion-free sheaves over $S$. Indeed, if $\mathcal{F}$ is such a sheaf, then its dual $\mathcal{F}^{*}$ (being reflexive) must be locally free, and the canonical inclusion $\mathcal{F} \hookrightarrow \mathcal{F}^{* *}$ has quotient $\mathcal{F}^{* *} / \mathcal{F}$ supported on a finite set of points, and hence has finite length, say $n$. Tensoring the inclusion $\mathcal{F} \hookrightarrow \mathcal{F}^{* *}$ with the line bundle $\mathcal{F}^{*}$, we obtain a sheaf of ideals $\mathcal{F} \otimes \mathcal{F}^{*} \subseteq \mathcal{O}_{S}$ of colength $n$, that is, a point of the Hilbert scheme $\mathrm{Hilb}_{n}(S)$. In this way we obtain a natural identification

$$
\mathcal{R}(S) \cong \operatorname{Pic} S \times \bigsqcup_{n \geq 0} \operatorname{Hilb}_{n}(S) ;
$$

the projection $\gamma_{S}: \mathcal{R}(S) \rightarrow$ Pic $S$ onto the first factor in (2.1) sends the class of $\mathcal{F}$ to the class of $\mathcal{F}^{* *}$ in Pic $S$. As we shall see more clearly below, this is analogous to the map $\gamma$ that we are studying in the noncommutative case.

In the case where $S$ is an affine surface, we may replace $\mathcal{F}$ by the corresponding module $F$ of global sections, and embed it as an ideal in $\mathcal{O}(S)$. The statements above then translate into statements about ideals in $\mathcal{O}(S)$ : in particular, an ideal $F$ has finite codimension in its bidual $F^{* *}$.

The map $\bar{\gamma}$. Let us specialize to the case 2 where $S=T^{*} X$ for some smooth affine curve $X$. In this case the projection $p: T^{*} X \rightarrow X$ induces an isomorphism $\operatorname{Pic} X \cong \operatorname{Pic}\left(T^{*} X\right)$ (see Remark 4.3): we define the map $\bar{\gamma}: \overline{\mathcal{R}} \rightarrow \operatorname{Pic} X$ to be the composition of the projection $\gamma_{S}$ above with this isomorphism. If $I$ is an ideal in $\mathcal{O}(X)$, then $p^{*}(I) \in \operatorname{Pic}\left(T^{*} X\right)$ is represented by the extended ideal $I \overline{\mathcal{D}} \subseteq \overline{\mathcal{D}} \equiv$ $\mathcal{O}\left(T^{*} X\right)$. Thus each invertible ideal $L \subseteq \overline{\mathcal{D}}$ is isomorphic to some $I \overline{\mathcal{D}}$, and $I$ is determined up to isomorphism by $L$. So the definition of $\bar{\gamma}$ amounts to the following: if $F$ is an ideal in $\overline{\mathcal{D}}$, then $\bar{\gamma}(F)$ is the unique ideal class $(I)$ such that $F^{* *}$ is isomorphic to I $\overline{\mathcal{D}}$.

The next Proposition gives several other ways to define $\bar{\gamma}$.

Proposition 2.1. Let $F$ be an ideal in $\overline{\mathcal{D}}$, and let $I$ be an ideal in $\mathcal{O}(X)$. Then the following are equivalent.

(i) $F$ and $I \overline{\mathcal{D}}$ determine the same class in the Grothendieck group $G_{0}\left(T^{*} X\right)$.

(ii) $F^{* *}$ and $I \overline{\mathcal{D}}$ determine the same class in the Grothendieck group $K_{0}\left(T^{*} X\right)$.

(iii) $F^{* *}$ and $I \overline{\mathcal{D}}$ determine the same class in $\operatorname{Pic}\left(T^{*} X\right)$.

(iv) There is an injective homomorphism $F \hookrightarrow I \overline{\mathcal{D}}$ with finite dimensional cokernel.

Proof. (i) $\Rightarrow$ (ii). Suppose (i) holds. Then the dual modules $F^{*}$ and $I^{*} \overline{\mathcal{D}}$ also determine the same class in $G_{0}\left(T^{*} X\right)$. Applying this remark again, we find that $F^{* *}$ and $I \overline{\mathcal{D}}$ determine the same class in $G_{0}\left(T^{*} X\right)$. Because $T^{*} X$ is smooth, the canonical map $K_{0}\left(T^{*} X\right) \rightarrow G_{0}\left(T^{*} X\right)$ is an isomorphism, so (ii) follows.

(ii) $\Rightarrow$ (iii). This is trivial, since det $: K_{0}\left(T^{*} X\right) \rightarrow \operatorname{Pic}\left(T^{*} X\right)$ maps the class of a line bundle to itself.

(iii) $\Rightarrow$ (iv). We have already seen that the canonical inclusion $F \hookrightarrow F^{* *}$ has finite dimensional cokernel, so this follows at once.

(iv) $\Rightarrow$ (i). Let $T$ be the cokernel of the given inclusion $F \hookrightarrow I \overline{\mathcal{D}}$, so that we

\footnotetext{
${ }^{2}$ More generally, in the parts of what follows that are not concerned with $\mathcal{D}, S$ could be the total space of any line bundle over $X$.
} 
have $[F]+[T]=[I \overline{\mathcal{D}}]$ in $G_{0}\left(T^{*} X\right)$. The support of $T$ has codimension 2, so (see Appendix A) $[T]$ lies in the kernel of the map

$$
\operatorname{rk} \oplus \operatorname{det}: G_{0}\left(T^{*} X\right) \cong K_{0}\left(T^{*} X\right) \rightarrow \mathbb{Z} \oplus \operatorname{Pic}\left(T^{*} X\right) \text {. }
$$

This map is an isomorphism (see Appendix A), hence $[T]=0$ in $G_{0}\left(T^{*} X\right)$ and (i) follows.

Comparison with the noncommutative case. Let $M$ be a rank 1 torsion-free (right) $\mathcal{D}$-module. We may embed $M$ as an ideal in $\mathcal{D}$ and give it the induced filtration; we write $\bar{M}:=$ gr $M$ for the associated graded $\overline{\mathcal{D}}$-module. If we change the embedding of $M$ in $\mathcal{D}$, the induced filtration changes at most by an overall shift, so the isomorphism class of $\bar{M}$ (forgetting the filtration) depends only on the isomorphism class of $M$. Thus we get a well-defined map gr $: \mathcal{R} \rightarrow \overline{\mathcal{R}}$, fitting into the diagram (1.1) in the Introduction. We can now give the

Proof of Theorem 1.2. Let $M$ be an ideal in $\mathcal{D}$. By definition, $\gamma(M)$ is the unique ideal class $(I) \in \operatorname{Pic} X$ such that $M$ is stably isomorphic to $I \mathcal{D}$. That means that for some $k \geq 0$, we have

$$
M \oplus \mathcal{D}^{k} \cong I \mathcal{D} \oplus \mathcal{D}^{k}
$$

Using the induced filtrations, the graded modules associated to the two sides of (2.2) are $\bar{M} \oplus \overline{\mathcal{D}}^{k}$ and $I \overline{\mathcal{D}} \oplus \overline{\mathcal{D}}^{k}$. Certainly, these modules are in general not isomorphic (because $\bar{M}$ is not projective); but Ginzburg's lemma (see Appendix B) implies that they determine the same class in $G_{0}\left(T^{*} X\right)$. By Proposition 2.1 ((i) $\Rightarrow$ (iii)), this means that $\bar{\gamma}(\bar{M})=(I)=\gamma(M)$.

\section{The ACtion of Pic $\mathcal{D}$}

In this section we prove (among other things) Theorems 1.1 and 1.3, We shall assume that $X$ is not isomorphic to $\mathbb{A}^{1}$ : in that case Theorem 1.1 is vacuous and Theorem 1.3 is already known. The reason for excluding $\mathbb{A}^{1}$ is that we shall need to use a theorem of Makar-Limanov (see (ML), which states that except in the case $X \cong \mathbb{A}^{1}$ the ring $\mathcal{O}(X) \subset \mathcal{D}$ is characterized as the unique maximal abelian ad-nilpotent subalgebra of $\mathcal{D}$.

We recall from [CH2] some information about $\mathrm{Pic} \mathcal{D}$. By definition, the elements of Pic $\mathcal{D}$ are the isomorphism classes of invertible $\mathcal{D}$-bimodules (with scalars $\mathbb{C} \subset \mathcal{D}$ acting the same way on both sides). Let $P$ be an invertible $\mathcal{D}$-bimodule. Then Cannings and Holland show that $P$ is isomorphic as left $\mathcal{D}$-module to $\mathcal{D} I$ for some ideal $I$ in $\mathcal{O}(X)$. The (commuting) structure of right $\mathcal{D}$-module is then determined by an isomorphism $\varphi: \mathcal{D} \rightarrow \operatorname{End}_{\mathcal{D}}(\mathcal{D} I)$ : we denote by $(\mathcal{D} I)_{\varphi}$ the $\mathcal{D}$-bimodule determined in this way by a pair $(I, \varphi)$.

Recall that $\mathbb{K}$ denotes the field of rational functions on $X$. In what follows, we shall identify $\operatorname{End}_{\mathcal{D}}(\mathcal{D} I)$ with the subring $I^{*} \mathcal{D} I$ of $\mathcal{D}\left(\mathbb{K}\right.$ ) (where $I^{*} \subset \mathbb{K}$ is the fractional ideal inverse to $I$ ): indeed, we have

$$
\begin{aligned}
\operatorname{End}_{\mathcal{D}}(\mathcal{D} I) & =\{\theta \in \mathcal{D}(\mathbb{K}): \mathcal{D} I \theta \subseteq \mathcal{D} I\} \\
& =\{\theta \in \mathcal{D}(\mathbb{K}): I \theta \subset \mathcal{D} I\} \\
& =I^{*} \mathcal{D} I .
\end{aligned}
$$

The isomorphism $\varphi: \mathcal{D} \rightarrow I^{*} \mathcal{D} I$ extends uniquely to an automorphism of $\mathcal{D}(\mathbb{K})$ (which we denote by the same symbol). By Makar-Limanov's theorem, $\varphi$ must map 
$\mathcal{O}(X)$ to itself, and hence also must preserve the filtration (by order of operator). We denote by $\sigma \in \operatorname{Aut}(\mathcal{O}(X))$ the restriction 3 of $\varphi$ to $\mathcal{O}(X)$. Theorem 1.1 follows trivially from the following explicit formula describing the (right) action of Pic $\mathcal{D}$ on $\mathrm{Pic} X$.

Proposition 3.1. Let $P:=(\mathcal{D} I)_{\varphi}$, and let $(J) \in$ Pic $X$. Then we have

$$
J . P=\sigma^{-1}(J I) \text {. }
$$

Proof. Note first that $J \mathcal{D} \otimes_{\mathcal{D}}(\mathcal{D} I)_{\varphi} \cong(J \mathcal{D} I)_{\varphi}$ (as right $\mathcal{D}$-modules); also, $\varphi$ defines (by restriction) an isomorphism of right $\mathcal{D}$-modules

$$
\varphi: \sigma^{-1}(J I) \mathcal{D} \rightarrow(J I)\left(I^{*} \mathcal{D} I\right)_{\varphi}=(J \mathcal{D} I)_{\varphi} .
$$

Since $\gamma: \mathcal{R} \rightarrow \operatorname{Pic} X$ is equivariant with respect to $\operatorname{Pic} \mathcal{D}$, we have indeed

$$
\begin{aligned}
J . P & =\gamma(J \mathcal{D}) . P \\
& =\gamma\left(J \mathcal{D} \otimes_{\mathcal{D}} P\right) \\
& =\gamma\left(\sigma^{-1}(J I) \mathcal{D}\right) \\
& =\sigma^{-1}(J I),
\end{aligned}
$$

as claimed.

Remark 3.2. Let Aut $\mathcal{D}$ be the group of $\mathbb{C}$-automorphisms of $\mathcal{D}$; then we have the homomorphism Aut $\mathcal{D} \rightarrow \operatorname{Pic} \mathcal{D}$ sending $\varphi \in$ Aut $\mathcal{D}$ to the bimodule $(\mathcal{D})_{\varphi}$. The kernel of this map is the group of inner automorphisms of $\mathcal{D}$, so the group Out $\mathcal{D}$ of outer automorphism classes can be regarded as a subgroup of Pic $\mathcal{D}$. These remarks hold for any $\mathbb{C}$-algebra $\mathcal{D}$; in our case it follows from Proposition 3.1 that the stabilizer of the neutral element of Pic $X$ is exactly Out $\mathcal{D}$, so we may identify the homogeneous space Out $\mathcal{D} \backslash \operatorname{Pic} \mathcal{D}$ with $\operatorname{Pic} X$.

Now, the group Aut $\mathcal{O}(X)$ acts (on the left) on $\mathcal{O}(X)$ and thence on Pic $X$, so we can form the semi-direct product $S:=\operatorname{Pic} X \rtimes \operatorname{Aut}(\mathcal{O}(X))$. The multiplication in $S$ is given by

$$
(I, \sigma)(J, \tau)=(I \sigma(J), \sigma \tau)
$$

Lemma 3.3 ([CH2 $)$. The man $(\mathcal{D} I)_{\varphi} \mapsto(I, \sigma)$ induces a well-defined homomorphism of groups from $\mathrm{Pic} \mathcal{D}$ onto $S$.

Sketch of proof. Recall that $\sigma:=\varphi \mid \mathcal{O}(X)$. The lemma follows from the fact that the multiplication of the bimodules $(\mathcal{D} I)_{\varphi}$ is given by

$$
(\mathcal{D} I)_{\varphi} \otimes_{\mathcal{D}}(\mathcal{D} J)_{\psi} \cong(\mathcal{D} I \sigma(J))_{\varphi \psi}
$$

The isomorphism is defined by $D_{1} \otimes_{\mathcal{D}} D_{2} \mapsto D_{1} \varphi\left(D_{2}\right)$. See [CH2 for more details.

The semi-direct product $S$ acts naturally (on the right) on $\overline{\mathcal{D}}$-modules $F$ by

$$
F .(I, \sigma)=\widehat{\sigma}_{*}\left(F \otimes_{\overline{\mathcal{D}}} I \overline{\mathcal{D}}\right),
$$

\footnotetext{
${ }^{3}$ Generically $\mathcal{O}(X)$ has no nontrivial automorphisms, so $\sigma$ is the identity.

${ }^{4}$ Cannings and Holland use the map $(\mathcal{D} I)_{\varphi} \mapsto\left(I^{*}, \sigma\right)$, but this differs from ours only by an automorphism of $S$.
} 
where $\widehat{\sigma}$ denotes the natural lifting of $\sigma$ to an automorphism of $\overline{\mathcal{D}} \equiv \mathcal{O}\left(T^{*} X\right)$. In the case where $F$ is an ideal in $\overline{\mathcal{D}}$ that is equivalent to

$$
F .(I, \sigma)=\widehat{\sigma}^{-1}(F I) .
$$

Thus $S$ acts naturally on the space of ideals $\overline{\mathcal{R}}$ : using Lemma 3.3, we may regard this as an action of $\mathrm{Pic} \mathcal{D}$. Theorem 1.3 will follow easily from the next

Proposition 3.4. The map gr: $\mathcal{R} \rightarrow \overline{\mathcal{R}}$ is equivariant with respect to the actions of $\operatorname{Pic} \mathcal{D}$.

To see this, we need one more lemma. The graded algebra associated to $I^{*} \mathcal{D} I$ is canonically identified with $\overline{\mathcal{D}}$, so that $\varphi$ induces a graded automorphism $\bar{\varphi}$ of $\overline{\mathcal{D}}$.

Lemma 3.5. We have $\bar{\varphi}=\widehat{\sigma}$.

Proof. Recall that $\overline{\mathcal{D}}$ is just the symmetric algebra of the module $\operatorname{Der} \mathcal{O}(X)$ of derivations of $\mathcal{O}(X)$. By construction, $\bar{\varphi}$ and $\widehat{\sigma}$ coincide on $\mathcal{O}(X)$, so we have only to see that they agree on $\operatorname{Der} \mathcal{O}(X)$. Now, the identification

$$
I^{*} \mathcal{D}_{\leq 1} I / \mathcal{O}(X) \rightarrow \overline{\mathcal{D}}_{1} \equiv \operatorname{Der} \mathcal{O}(X)
$$

sends an operator $D$ to the derivation $f \mapsto[D, f]$. Also, if $\partial \in \operatorname{Der} \mathcal{O}(X)$ and $f \in \mathcal{O}(X)$, then in $\mathcal{D}$ we have the relation $[\partial, f]=\partial(f)$, whence

$$
\begin{aligned}
{[\varphi(\partial), f] } & =\left[\varphi(\partial), \varphi\left(\sigma^{-1}(f)\right)\right] \\
& =\varphi\left[\partial, \sigma^{-1}(f)\right] \\
& =\varphi\left(\partial\left(\sigma^{-1}(f)\right)\right) \\
& =\left(\sigma \partial \sigma^{-1}\right)(f) .
\end{aligned}
$$

Combining these two remarks, we find that $\bar{\varphi}(\partial)=\sigma \partial \sigma^{-1}$, which (by definition) is indeed $\widehat{\sigma}(\partial)$.

Now we can give the

Proof of Proposition 3.4. Let $M$ be an ideal in $\mathcal{D}$, and let $P=(\mathcal{D} I)_{\varphi}$ be an invertible $\mathcal{D}$-bimodule, as above. Then $M \otimes_{\mathcal{D}} P=M I$ with the structure of (right) $\mathcal{D}$-module determined via the isomorphism $\varphi$. Since $\varphi$ is filtration-preserving, it follows that $\overline{M \otimes_{\mathcal{D}} P}=\bar{M} I$ with the structure of $\overline{\mathcal{D}}$-module determined via $\bar{\varphi}$; that is, using Lemma 3.5

$$
\operatorname{gr}\left(M \otimes_{\mathcal{D}} P\right) \equiv \overline{M \otimes_{\mathcal{D}} P} \cong \widehat{\sigma}^{-1}(\bar{M} I) .
$$

Comparing with (3.2), we see that the map gr is equivariant.

Proof of Theorem 1.3. It is clear that if $F$ is an ideal in $\overline{\mathcal{D}}$ then the action (3.2) of $S$ preserves the codimension of $F$ in $F^{* *}$, so Theorem 1.3 follows at once from Proposition 3.4

Remark. Comparing Proposition 3.1 with the formula (3.2) shows that our map $\bar{\gamma}: \overline{\mathcal{R}} \rightarrow \operatorname{Pic} X$ is equivariant with respect to the actions of $S$ (which we may regard as actions of Pic $\mathcal{D}$ ). Thus all the maps in the commutative diagram (1.1) are equivariant with respect to the actions of $\mathrm{Pic} \mathcal{D}$. 


\section{FAT IDEALS}

Following $[\mathrm{BGK}]$, we call an ideal in $\mathcal{D}$ or $\overline{\mathcal{D}}$ fat if its intersection with $\mathcal{O}(X)$ is nonzero. The usefulness of this notion comes from the simple

Lemma 4.1. Every ideal in $\mathcal{D}$ or $\overline{\mathcal{D}}$ is isomorphic to a fat one.

Proof. We give the proof for $\overline{\mathcal{D}}$ : the proof for $\mathcal{D}$ is the same (see St, Lemma 4.2). Recall again that $\mathbb{K}$ is the quotient field of $\mathcal{O}(X)$. The algebra $\mathbb{K} \otimes_{\mathcal{O}(X)} \overline{\mathcal{D}}$ is a principal ideal domain (because it is a polynomial algebra $\mathbb{K}[\xi]$ : for $\xi$ we can take any nonzero derivation of $\mathbb{K})$. Given an ideal $F \subseteq \overline{\mathcal{D}}$, let $a$ be a generator of its extension to $\mathbb{K} \otimes_{\mathcal{O}(X)} \overline{\mathcal{D}}$. Then the fractional ideal $a^{-1} F$ has nonzero intersection with $\mathbb{K}$; multiplying by a suitable element of $\mathcal{O}(X)$ we get a fat (integral) ideal isomorphic to $F$.

Lemma 4.2. Let $L$ be an invertible fat ideal in $\overline{\mathcal{D}}$, and let $I:=L \cap \mathcal{O}(X)$. Then $L=I \overline{\mathcal{D}}$.

Proof. Clearly, $I \overline{\mathcal{D}} \subseteq L$. The divisor of zeros of $I \overline{\mathcal{D}}$ is a linear combination of fibres of the projection $p: T^{*} X \rightarrow X$; the divisor of zeros of $L$ must be smaller, that is, $L=J \overline{\mathcal{D}}$ for some $J \supseteq I$. But then $L \cap \mathcal{O}(X)=J$, so $J=I$.

Remark 4.3. We have essentially given a proof of the fact (which we used earlier) that the map $p^{*}: \operatorname{Pic} X \rightarrow \operatorname{Pic}\left(T^{*} X\right)$ is an isomorphism. Indeed, $p^{*}$ is obviously injective, because if $i: X \rightarrow E$ is the inclusion of the zero section, then $p i=\operatorname{Id}_{X}$, so $i^{*} p^{*}$ is the identity on Pic $X$. Lemmas 4.1 and 4.2 show that $p^{*}$ is surjective.

Proposition 4.4. Let $F$ be a fat ideal in $\overline{\mathcal{D}}$. Then there is a unique ideal $I$ in $\mathcal{O}(X)$ such that $F$ has finite codimension in $I \overline{\mathcal{D}}$. Furthermore, $\bar{\gamma}(F)=(I)$.

Proof. We may realize the dual module $F^{*}$ of $F$ as the fractional ideal

$$
F^{*}=\{f \in \operatorname{Frac}(\overline{\mathcal{D}}): f F \subseteq \overline{\mathcal{D}}\} .
$$

With this understanding, the map $F \mapsto F^{*}$ reverses inclusions, and the canonical inclusion $F \hookrightarrow F^{* *}$ becomes an inclusion of ideals in $\overline{\mathcal{D}}$. If $F$ is fat, so is $F^{* *}$, so Lemma 4.2 gives $F^{* *}=I \overline{\mathcal{D}}$, where $I:=F^{* *} \cap \mathcal{O}(X)$. This shows existence of the ideal $I$ in the proposition. To see uniqueness, suppose that also $F \subseteq J \overline{\mathcal{D}}$ with finite codimension. Taking biduals, we get $F \subseteq F^{* *}=I \overline{\mathcal{D}} \subseteq J \overline{\mathcal{D}}$. Thus $I \subseteq J$ and $J \overline{\mathcal{D}} / I \overline{\mathcal{D}}$ has finite dimension, which is impossible unless $I=J$. Finally, the last assertion in the Proposition comes from the fact that $\bar{\gamma}$ can be defined by the property (iv) in Proposition 2.1.

Clearly, if $M$ is fat, so is $\bar{M}$. So Proposition 4.4 implies:

Theorem 4.5. Let $M \subseteq \mathcal{D}$ be a fat ideal. Then there is a unique ideal $I$ in $\mathcal{O}(X)$ such that $\bar{M}$ has finite codimension in $I \overline{\mathcal{D}}$. Furthermore, $I \overline{\mathcal{D}}=\bar{M}^{* *}$, and $\gamma(M)=(I)$ in Pic $X$.

\section{The Cannings-Holland CORRespondence}

Recall from [CH1] that a subspace $V \subseteq \mathcal{O}(X)$ is called primary (more precisely, $x$-primary) if it contains a power of the maximal ideal $\mathfrak{m}_{x}$ of functions that vanish at a point $x \in X$ (we write $V=V_{x}$ in this case). Now, $V \subseteq \mathcal{O}(X)$ is called primary decomposable if it is an intersection of primary subspaces $V_{x}$ with $V_{x}=\mathcal{O}(X)$ for 
almost all $x \in X$. By CH1, Theorem 2.4, the primary decomposition of $V$ is uniquely determined. If $V$ is a primary decomposable subspace of $\mathcal{O}(X)$, we set

$$
M_{V}:=\{D \in \mathcal{D}: D \cdot \mathcal{O}(X) \subseteq V\}
$$

(here $D$.f denotes the function obtained by letting $D$ act on $f$, not to be confused with the operator $D f \in \mathcal{D}$ ). Clearly, $M_{V}$ is a right ideal in $\mathcal{D}$. The main result of [CH1] was the following.

Theorem 5.1. The map $V \mapsto M_{V}$ is a 1-1 correspondence between the lattice of primary decomposable subspaces of $\mathcal{O}(X)$ and the lattice of fat right ideals in $\mathcal{D}$. The inverse map is given by $M \mapsto M . \mathcal{O}(X)$.

Theorem 4.5 associates to each fat ideal $M \subseteq \mathcal{D}$ an ideal $I$ in $\mathcal{O}(X)$ (namely, the ideal such that $\left.\bar{M}^{* *}=I \overline{\mathcal{D}}\right)$. If $V$ is a primary decomposable subspace of $\mathcal{O}(X)$ we write $I_{V}$ for the ideal associated in this way to $M_{V}$. The relationship of this construction to Theorem 5.1 is as follows.

Theorem 5.2. Let $V$ be the intersection of $x$-primary subspaces $V_{x}$, and let $n_{x}$ be the codimension of $V_{x}$ in $\mathcal{O}(X)$. Then we have

$$
I_{V}=\prod_{x \in X} \mathfrak{m}_{x}^{n_{x}}
$$

The proof rests on the following lemma.

Lemma 5.3. Let $V \subseteq \mathcal{O}(X)$ be primary decomposable, $I_{V} \subseteq \mathcal{O}(X)$ the corresponding ideal. Then

$$
\operatorname{dim}(\mathcal{O} / V)=\operatorname{dim}\left(\mathcal{O} / I_{V}\right)
$$

Proof. We start with the exact sequence of $\overline{\mathcal{D}}$-modules

$$
0 \rightarrow I_{V} \overline{\mathcal{D}} / \bar{M}_{V} \rightarrow \overline{\mathcal{D}} / \bar{M}_{V} \rightarrow \overline{\mathcal{D}} / I_{V} \overline{\mathcal{D}} \rightarrow 0 .
$$

Recall from [SS that the 1-length of a $\overline{\mathcal{D}}$-module is the maximum length of a chain of submodules such that the successive quotients are infinite-dimensional (over $\mathbb{C}$ ). Since the 1-length is additive, and the first term in (5.3) has finite dimension (and hence 1-length 0 ), we get

$$
\text { 1-length }\left(\overline{\mathcal{D}} / \bar{M}_{V}\right)=1 \text {-length }\left(\overline{\mathcal{D}} / I_{V} \overline{\mathcal{D}}\right) \text {. }
$$

By [SS], Lemma 3.10, this is equivalent to

$$
\text { length }\left(\mathcal{D} / M_{V}\right)=\operatorname{length}\left(\mathcal{D} / I_{V} \mathcal{D}\right) .
$$

By [CH1], Corollary 3.8, this in turn is equivalent to (5.2).

Now we can give the proof of Theorem 5.2, To simplify the notation, if $V_{x}$ is $x$-primary, we shall write simply $M_{x}$ and $I_{x}$ for the associated ideals in $\mathcal{D}$ and in $\mathcal{O}(X)$.

Proof of Theorem 5.2. We consider first the case where $V \equiv V_{x}$ is $x$-primary: thus $\mathfrak{m}_{x}^{N} \subseteq V_{x}$ for some $N$; it follows that $\mathfrak{m}_{x}^{N} \mathcal{D} \subseteq M_{x}$. Passing to the associated graded modules, we get

$$
\mathfrak{m}_{x}^{N} \overline{\mathcal{D}} \subseteq \bar{M}_{x} \subseteq \bar{M}_{x}^{* *}=I_{x} \overline{\mathcal{D}}
$$

and hence $\mathfrak{m}_{x}^{N} \subseteq I_{x}$. By the uniqueness of factorization of ideals in the Dedekind domain $\mathcal{O}(X)$, this means that $I_{x}=\mathfrak{m}_{x}^{k}$ for some $k \leq N$. Lemma 5.3 then 
gives that $n_{x}=k$; that is, we have proved Theorem 5.2 in the case where $V$ is $x$-primary.

In general, suppose that $V$ is the intersection of primary subspaces $V_{x}$; then we have $V \subseteq V_{x}$ for each $x$. It follows that $M_{V} \subseteq M_{x}$, and hence $\bar{M}_{V} \subseteq \bar{M}_{x}$. Taking biduals (inside the field of fractions of $\overline{\mathcal{D}}$, as in the proof of Theorem 4.4) we get $I_{V} \overline{\mathcal{D}} \subseteq I_{x} \overline{\mathcal{D}}$, and hence $I_{V} \subseteq I_{x}=\mathfrak{m}_{x}^{n_{x}}$. This means that if the factorization of $I_{V}$ is given by $I_{V}=\prod \mathfrak{m}_{x}^{k_{x}}$, then $n_{x} \leq k_{x}$ for all $x$. But by [CH1, Theorem 2.4, the left hand side of (5.2) is equal to $\sum n_{x}$, so Lemma 5.3 says that $\sum n_{x}=\sum k_{x}$. It follows that $n_{x}=k_{x}$ for all $x$.

Theorem 5.2 is in essence a more precise version of Theorem 1.4 from the Introduction. To see that, note first that the fat representative of an ideal class in $\mathcal{R}$ is unique up to multiplication by a rational function; that is, $\mathcal{R}$ is the quotient of the space of fat ideals by the equivalence relation 5

$$
M_{1} \sim M_{2} \Longleftrightarrow f M_{1}=g M_{2} \text { for some } f, g \in \mathcal{O}(X) .
$$

Obviously, if $f \in \mathcal{O}(X)$, then Theorem 5.1 makes $f V$ correspond to $f M_{V}$; also, if $M$ is a fat ideal, then $\overline{f M}=f \bar{M}$; hence for any primary decomposable $V$ we have $I_{f V}=f I_{V}$. In the usual correspondence between ideals and divisors, the ideal $I_{V}$ in (5.1) corresponds to the divisor $\operatorname{Div}(V)$ in (1.2). It follows that we have

$$
\operatorname{Div}(f V)=\operatorname{Div}(V)-\operatorname{Div}(f)
$$

(where $\operatorname{Div}(f)$ is the divisor of zeros of $f$ ). Thus (as claimed in the Introduction) the map $V \mapsto \operatorname{Div}(V)$ yields a well-defined map $\operatorname{Div}: \mathcal{R} \rightarrow \operatorname{Pic} X$. That this map coincides with $\gamma$ follows from Theorem 4.5 .

\section{Appendix A. $S K_{0}$ OF ALgebraic VARIETIES}

Here we provide proofs of a couple of (well known) facts for which we do not know a convenient reference. We need these facts only for affine varieties $X$; however, they are valid for any smooth quasi-projective variety, indeed, for any regular Noetherian scheme. We denote by $K_{0}(X)$ the Grothendieck group of vector bundles over $X$; in the affine case this is the same as the Grothendieck group of finitely generated projective $\mathcal{O}(X)$-modules. Taking the determinant of a vector bundle induces a map det $: K_{0}(X) \rightarrow$ Pic $X$. Considering also the rank of a vector bundle, we obtain a map (obviously surjective)

$$
\operatorname{rk} \oplus \operatorname{det}: K_{0}(X) \rightarrow \mathbb{Z} \oplus \operatorname{Pic} X \text {. }
$$

The kernel of this map is denoted by $S K_{0}(X)$. If $X$ is a curve, then $S K_{0}(X)=0$ (see, for example, $[\mathrm{H}]$, Ch. II, Ex. 6.11). In the proof of Proposition 2.1 we used also the fact that if $X$ is a curve, then $S K_{0}\left(T^{*} X\right)=0$. That is a special case of the following.

Proposition A.1. Let $E$ be the total space of a vector bundle over a smooth variety $X$. Then $S K_{0}(E)=0$ if and only if $S K_{0}(X)=0$.

\footnotetext{
${ }^{5}$ Our exposition would be smoother at this point if we worked with fractional ideals in $\mathcal{D}(\mathbb{K})$.
} 
Proof. Let $p: E \rightarrow X$ be the projection. Then we have a commutative diagram

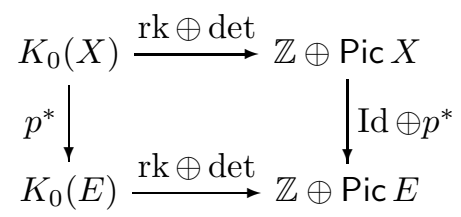

in which the two horizontal arrows are surjective. By [B], Theorem 3.2, p. 636 in the affine case, or Q , Proposition 4.1, p. 128 in general, the left hand vertical arrow $p^{*}$ is an isomorphism. It follows from the commutativity of the diagram (A.2) that the right hand vertical arrow is surjective, hence it too is an isomorphism (since it is obviously injective, cf. Remark 4.3). The Proposition is now obvious.

As well as $K_{0}(X)$ we have the Grothendieck group $G_{0}(X)$ of coherent sheaves over $X$; in the affine case this is the same as the Grothendieck group of finitely generated $\mathcal{O}(X)$-modules. For smooth $X$, the natural map $K_{0}(X) \rightarrow G_{0}(X)$ is an isomorphism (see, for example, Q, p. 124), so we may identify these two groups. We then have another important fact about $S K_{0}(X)$ : it is generated by the classes (in $G_{0}(X) \cong K_{0}(X)$ ) of sheaves whose support has codimension $\geq 2$. Here is a proof of the part of that which we used.

Proposition A.2. Let $\mathcal{F}$ be a coherent sheaf over $X$ whose support has codimension $\geq 2$. Then $[\mathcal{F}] \in S K_{0}(X)$.

Proof. Obviously, the rank of $\mathcal{F}$ is zero, so we have only to consider its determinant. Choose a resolution

$$
0 \rightarrow P_{n} \rightarrow \ldots \rightarrow P_{1} \rightarrow P_{0} \rightarrow \mathcal{F} \rightarrow 0
$$

of $\mathcal{F}$ by vector bundles $P_{i}$. By definition, $\operatorname{det} \mathcal{F}$ is the alternating product of the determinant line bundles of the $P_{i}$. This line bundle is trivial off the support of $\mathcal{F}$, that is, it has a rational section whose divisor of zeros and poles is contained in the support of $\mathcal{F}$. Since the support has codimension greater than 1 , that is impossible unless the divisor of zeros and poles is empty, that is, $\operatorname{det} \mathcal{F}$ is trivial, as claimed.

\section{Appendix B. A lemma of Ginzburg on filtrations}

Let $A$ be a positively filtered associative ring such that $\bar{A}:=\operatorname{gr} A$ is right Noetherian. Let $G_{0}(\bar{A})$ be the Grothendieck group of finitely generated right $\bar{A}$-modules, and let $M$ be a finitely generated right $A$-module equipped with a good filtration (recall that good means that the associated graded $\bar{A}$-module $\bar{M}$ is finitely generated). As usual, we write $[\bar{M}]$ for the class of $\bar{M}$ in $G_{0}(\bar{A})$. The following observation is (apparently) due to Ginzburg (see G], Corollary 1.3, p. 338).

Lemma B.1. $[\bar{M}]$ does not depend on the choice of good filtration on $M$.

Proof. Call two filtrations $M_{\bullet}$ and $M_{\bullet}^{\prime}$ on $M$ adjacent if $M_{k-1} \subseteq M_{k}^{\prime} \subseteq M_{k}$ for all $k \in \mathbb{Z}$. Given a pair of adjacent filtrations, set $\bar{L}:=\bigoplus_{k \in \mathbb{Z}} M_{k}^{\prime} / M_{k-1}$ and $\bar{N}:=\bigoplus_{k \in \mathbb{Z}} M_{k} / M_{k}^{\prime}$. Then we have the obvious exact sequences of (graded) $\bar{A}$ modules:

$$
0 \rightarrow \bar{L} \rightarrow \bar{M} \rightarrow \bar{N} \rightarrow 0 \quad \text { and } \quad 0 \rightarrow \bar{N}(-1) \rightarrow \bar{M}^{\prime} \rightarrow \bar{L} \rightarrow 0 .
$$


Forgetting the grading, we have $\bar{N}(-1) \cong \bar{N}$, and therefore

$$
[\bar{M}]=[\bar{L}]+[\bar{N}]=[\bar{L}]+[\bar{N}(-1)]=\left[\bar{M}^{\prime}\right] \quad \text { in } G_{0}(\bar{A}) .
$$

Now, for any filtrations $M_{\bullet}$ and $M_{\bullet}^{\prime}$ on $M$, define a sequence of adjacent filtrations $\left\{M_{\bullet}^{(j)}: j \in \mathbb{Z}\right\}$ by $M_{k}^{(j)}:=M_{k}+M_{k-j}^{\prime}$ so that $M_{k-1}^{(j)} \subseteq M_{k}^{(j+1)} \subseteq M_{k}^{(j)}$ for all $k, j \in \mathbb{Z}$. If $M_{\bullet}$ and $M_{\bullet}^{\prime}$ are both good, there is an $n \in \mathbb{N}$ such that $M_{k-n}^{\prime} \subseteq$ $M_{k} \subseteq M_{k+n}^{\prime}$ for all $k \in \mathbb{Z}$ (see, for example, Bj], Proposition 1.15, p. 458). Thus, in the case of good filtrations we have $M_{\bullet}^{(j)}=M_{\bullet}^{\prime}$ for $j \leq-n$ and $M_{\bullet}^{(j)}=M_{\bullet}$ for $j \geq n$, so the lemma follows from (B.1).

\section{REFERENCES}

[BGK] V. Baranovsky, V. Ginzburg and A. Kuznetsov, Wilson's Grassmannian and a noncommutative quadric, Internat. Math. Res. Notices 21 (2003), 1155-1197.

[B] H. Bass, Algebraic K-theory, W. A. Benjamin Inc., New York-Amsterdam, 1968.

[BN] D. Ben-Zvi and T. Nevins, Perverse bundles and Calogero-Moser spaces, preprint arXiv: math.AG/0610097.

[Be] Yu. Berest, Calogero-Moser spaces over algebraic curves, Selecta Math. (to appear), arXiv:0809.4521

[BC] Yu. Berest and O. Chalykh, Ideals of rings of differential operators on algebraic curves (with an appendix by George Wilson), arXiv:0809.3258

[BW1] Yu. Berest and G. Wilson, Automorphisms and ideals of the Weyl algebra, Math. Ann. 318 (2000), 127-147.

[BW2] Yu. Berest and G. Wilson, Ideal classes of the Weyl algebra and noncommutative projective geometry (with an Appendix by M. Van den Bergh), Internat. Math. Res. Notices 26 (2002), $1347-1396$.

[Bj] J.-E. Björk, Analytic D-modules, Mathematics and its Applications 247, Kluwer Acad. Publ., Dordrecht, 1993,

[CH1] R. C. Cannings and M. P. Holland, Right ideals of rings of differential operators, J. Algebra 167 (1994), 116-141.

[CH2] R. C. Cannings and M. P. Holland, Etale covers, bimodules and differential operators, Math. Z. 216 (1994), 179-194.

[G] V. Ginzburg, Characteristic varieties and vanishing cycles, Invent. Math. 84 (1986), 327-402.

[H] R. Hartshorne, Algebraic Geometry, Springer-Verlag, 1977.

[ML] L. Makar-Limanov, Rings of differential operators on algebraic curves, Bull. London Math. Soc. 21 (1989), 538-540.

[OSS] C. Okonek, M. Schneider and H. Spindler, Vector bundles on complex projective spaces, Birkhäuser, Boston, Mass., 1980.

[Q] D. Quillen, Higher algebraic K-theory. I, Lecture Notes in Math. 341, Springer, Berlin 1973, pp. 85-147.

[SS] S. P. Smith and J. T. Stafford, Differential operators on an affine curve, Proc. London Math. Soc. (3) $\mathbf{5 6}$ (1988), 229-259.

[St] J. T. Stafford, Endomorphisms of right ideals in the Weyl algebra, Trans. Amer. Math. Soc. 299 (1987), 624-639.

[W1] G. Wilson, Bispectral commutative ordinary differential operators, J. Reine Angew. Math. 442 (1993), 177-204.

[W2] G. Wilson, Collisions of Calogero-Moser particles and an adelic Grassmanian (with an Appendix by I. G. Macdonald), Invent. Math. 133 (1998), 1-41.

[W3] G. Wilson, The adelic Grassmannian of an algebraic curve, in preparation.

Department of Mathematics, Cornell University, Ithaca, NY 14853, USA

E-mail address: berest@math.cornell.edu

Mathematical Institute, 24-29 St Giles, Oxford OX1 3LB, UK

E-mail address: wilsong@maths.ox.ac.uk 